\title{
miR-21 protects against lipopolysaccharide-stimulated acute kidney injury and apoptosis by targeting CDK6
}

\author{
Wei Wei ${ }^{1}$, Yuan-Yuan Yao $^{2}$, Hong-Yuan $\mathrm{Bi}^{1}$, Zhe Zhai ${ }^{1}$, Yan Gao ${ }^{1}$ \\ ${ }^{1}$ Department of Intensive Care Unit, The Fourth Affiliated Hospital of Harbin, Medical University, Harbin 150080 , China; ${ }^{2}$ Department of General \\ Internal Medicine, Harbin Red Cross Center Hospital, Harbin 150080, China \\ Contributions: (I) Conception and design: W Wei, Y Gao; (II) Administrative support: W Wei, YY Yao; (III) Provision of study materials or patients: \\ W Wei, YY Yao, Z Zhai, HY Bi; (IV) Collection and assembly of data: W Wei, Z Zhai; (V) Data analysis and interpretation: W Wei, Z Zhai, HY Bi, \\ Y Gao; (VI) Manuscript writing: All authors; (VII) Final approval of manuscript: All authors. \\ Correspondence to: Yan Gao. Department of Intensive Care Unit, The Fourth Affiliated Hospital of Harbin, Medical University, Harbin 150080, \\ China. Email: gaoyan_icu@163.com.
}

\begin{abstract}
Background: Septic acute kidney injury (AKI) causes a sharp deterioration of renal function, and it is a major reason for mortality in intensive care units. Although miR-21 has been proven to be dysregulated in patients with sepsis, the evidence is scarce concerning its role in mediating cellular apoptosis in AKI.

Methods: Mice were injected intraperitoneally with $10 \mathrm{mg} / \mathrm{kg}$ of lipopolysaccharide (LPS) to establish septic AKI model, miR-21 mimic and inhibitor were used to manipulate the expression of miR-21, the creatinine levels were detected by a creatinine assay kit, the renal cell proliferation and apoptosis were detected by MTT assay, flow cytometry assay and acridine orange/ethidium bromide (AO/EB) fluorescence staining, the renal function was evaluated by renal histology and tubular injury score, western blot analysis was used to detect the target protein levels. Several bioinformatics tools were performed to show the downstream target of miR-21, and further confirmed by luciferase reporter assay and caspase-3 activity assay. Results: miR-21 silencing was able to promote renal function and decrease LPS-stimulated renal cell apoptosis in vitro and in vivo, and it could decrease the Bax/Bcl-2 ratio and caspase- 3 activity. On the contrary, miR-21 overexpression had the opposite effects. Cyclin-dependent kinase 6 (CDK6) was confirmed as a target gene of miR-21 and was associated with renal cell apoptosis. Moreover, miR-21 was also found to be up-regulated in septic AKI.
\end{abstract}

Conclusions: Current evidences suggest that miR-21 has a potential application in treating septic AKI.

Keywords: miRNA; apoptosis; acute kidney injury (AKI); cyclin-dependent kinase 6 (CDK6)

Submitted Jun 06, 2019. Accepted for publication Feb 05, 2020.

doi: $10.21037 /$ atm.2020.03.01

View this article at: http://dx.doi.org/10.21037/atm.2020.03.01

\section{Introduction}

The definition of sepsis has traditionally been defined as a systemic inflammatory response syndrome occurring concurrently with suspected or documented infection $(1,2)$. The septic acute kidney injury (AKI) develops commonly in the cases of the intensive care unit, it consumes large amounts of resources, and causes higher mortality rates when compared to other critical care conditions (3-5). Mortality rates in AKI triggered by sepsis are extremely high (6). While the overall AKI mortality rate lies at $45 \%$, patients with septic AKI suffer from a 70\% mortality rate $(7,8)$. Given that septic AKI is a commonly encountered clinical condition, there is a need to fully understand the underlying pathophysiologic pathways to formulate more effective therapies. In our previous work, we confirmed increased miR-21 in the serum of lipopolysaccharide (LPS)stimulated sepsis patients with low survival rate of AKI patients (9). Herein, the effect role of miR-21 in AKI and 
Page 2 of 12

Table 1 The general information of the septic AKI and controls

\begin{tabular}{lcc}
\hline Characteristics & Control & AKI III \\
\hline Number of patients & 23 & 53 \\
Sex (male/female) & $16 / 7$ & $25 / 28$ \\
Age (range in years) & 38 to 66 & 31 to 72 \\
Creatinine clearance rate $(\mu \mathrm{mol} / \mathrm{L})$ & $83.6 \pm 13.8$ & $436 \pm 63$ \\
\hline
\end{tabular}

its potential mechanism is still largely unclear.

MicroRNAs (miRs) are vital mediators of posttranscriptional gene regulation, which are involved in cellular differentiation, proliferation, migration, apoptosis, and aging $(10,11)$. Several studies have suggested that some miRs were dysregulated in AKI, such as miR-188, -30a, $-155,-18 \mathrm{a}$, and $-30 \mathrm{e}$, however, the potential mechanism is unclear (12). miR-21 displays potent anti-apoptotic properties, demonstrating abilities to halt apoptosis and enhance proliferation while contributing to the cellular mechanisms of tissue healing and renal damage (13-15). In the model of I/R-induced AKI, Song et al. confirmed that miR-21 can inhibit renal injury via inhibiting epithelial cell apoptosis and dendritic cell maturation (16). Nevertheless, there is still more information that can be acquired regarding miR-21's role in septic AKI.

In our study, we attempted to further elucidate the impacts of miR-21 in septic AKI in vitro and in vivo along with its underlying mechanism. Our findings concerning the relationship between miR-21 and cyclin-dependent kinase 6 (CDK6) in AKI may provide new pharmacological insights that may propel the development of clinical therapeutics.

\section{Methods}

\section{Subjects}

Fifty-three AKI patients were collected from Department of Intensive care unit, The Fourth Affiliated Hospital of Harbin Medical University between March 2015 and May 2018. The patients were included only if they were diagnosed as AKI III according to the KDIGO Clinical Practice Guidelines (17). In addition, 23 healthy human volunteers used as standard (healthy control). This work was approved by the Ethics Committee of Harbin Medical University, representatives of the subjects of the study signed informed consent forms. Table 1 shown the general information obtained from AKI patients and controls.

\section{Experimental reagents and cell cultures}

Bovine serum albumin (BSA) and LPS were bought from Sigma-Aldrich Chemical Corporation (MO, USA). DMEM medium and fetal bovine serum (FBS) were purchased from Gibco Corporation (CA, USA). Bcl-2, Bax and CDK6 were obtained from Cell Signaling Technology (MA, USA), while the caspase- 3 activity kit was purchased from Beyotime (Nantong, China). A creatinine assay kit was obtained from Abcom (CA, USA) to measure the serum creatinine levels. All remaining analytical grade reagents were bought from Sigma Chemical Corporation. HK-2 cells, obtained from American Type Culture Collection (MA, USA), were cultured in DMEM with $10 \% \mathrm{FBS}, 4 \mathrm{mM}$ L-glutamine, $0.15 \%$ sodium bicarbonate, $100 \mathrm{U} / \mathrm{mL}$ penicillin and $100 \mathrm{U} / \mathrm{mL}$ streptomycin (Sigma-Aldrich, MO, USA) at $37^{\circ} \mathrm{C}$ containing $5 \% \mathrm{CO}_{2}$.

\section{Animals}

Twenty-eight male C57BL6/J mice $(20 \pm 2 \mathrm{~g})$ aging 10 12 weeks were raised under standard conditions. All the animals' studies were agreed by the Model Animal Research Center of Harbin Medical University. In AKI group ( $\mathrm{n}=14)$, $10 \mathrm{mg} / \mathrm{kg}$ LPS was intraperitoneally injected to establish septic AKI model, while an equal volume of saline was given in control mice $(\mathrm{n}=14)$. Ethical approval was obtained from the Science and Technology Department of Heilongjiang Province. All animals were raised based on the Provisions and General Recommendation of the Chinese Experimental Animals Administration Legislation.

\section{Co-culture of miR-21 mimics or inbibitors in mice and cells}

The miR-21 mimics and inhibitors (Ribobio, Guangzhou, China) were employed to assess the impacts of miR-21 on LPS-stimulated AKI. In brief, miR-21 inhibitors (10 nmol) or miR-21 mimic ( $20 \mathrm{nmol})$ in $50 \mu \mathrm{L}$ PBS were administered into cauda veins once for 3 days to conduce miR-21 knockdown, normal saline was used as control, following LPS post-stimulation.

\section{MTT assay and flow cytometry (FCM) assay}

MTT assay was performed to measure cellular proliferation; $10^{4} \mathrm{HK}-2$ cells per well were subjected to $24 \mathrm{~h}$ incubation with serum-free medium. Subsequently, 
different concentrations of miR-21 mimics or inhibitors were co-cultured in HK-2 cells for next $48 \mathrm{~h}$. Following this, the cells were stimulated for next $24 \mathrm{~h}$ with LPS $(1 \mu \mathrm{g} / \mathrm{mL})$ or without. In control cells, no mimics and inhibitors of miR-21 and LPS were added. $20 \mu \mathrm{L}$ of MTT $(5 \mathrm{mg} / \mathrm{mL})$ was finally co-cultured in 96-well plate per well for $4 \mathrm{~h}$ co-incubation at $37^{\circ} \mathrm{C}$, then $150 \mu \mathrm{L}$ DMSO was added into each well after removing the supernatant. The optical density (OD) was measured at $570 \mathrm{~nm}$ wavelength using a microplate reader (Bio-Tek, GA, USA). For FCM assay, HK-2 cells after co-cultured with dysregulate miR-21 were collected, stained using Annexin V-FITC/PI Apoptosis Detection Kit (Vazyme, Nanjing, China), and analyzed by BD FACS Canto II flow cytometry (BD Biosciences, NJ, USA).

\section{Renal bistology and tubular injury score}

Renal tissues that were previously fixed with formalin and embedded with paraffin were frozen-sectioned at a thickness of $3 \mu \mathrm{m}$, hematoxylin and eosin (HE)-stained, and observed using an optical microscope (Olympus Optical, Tokyo, Japan). The scoring criteria of tubular injury includes: $0=$ normal tubular histology; 1 = nuclear condensation, brush border loss, tubular cell swelling, with up to $1 / 3$ of tubular profiles showing nuclear loss; $2=$ as for score 1,116 but nuclear loss seen in $1 / 3-2 / 3$ of renal tubules; and $3=$ nuclear loss seen in more 117 profiles and more than $2 / 3$ of renal tubules.

\section{Acridine orange/ethidium bromide (AO/EB) fluorescence staining}

miR-21 mimics or inhibitors were used to transfect HK-2 $\left(2 \times 10^{4}\right)$ cells. The cells were left to stand for 5 minutes with mixed solution containing $20 \mu \mathrm{L}$ acridine orange and 20 $\mu \mathrm{L}$ ethidium bromide (Solarbio of Biotechnology, Beijing, China). The morphology of HK-2 cells were detected using a fluorescence microscopy (ThernoFisher scientific, USA). Apoptotic Rate (\%) = number of apoptotic cells/total number of cells.

\section{qRT-PCR}

Total RNA from renal tissues and cells were extracted using a TRIzol RNA extraction kit (Invitrogen, CA, USA). The extracted RNA was then reverse-transcribed to cDNA using an iScript cDNA Synthesis Kit (Bio-Rad,
CA, USA). miR-21 expression levels were quantified with the Bulge-Loop miRNA qPCR Primer Set (RiboBio, Guangzhou, China) in addition to the Takara SYBR mastermix Kit (Bio-Rad, CA, USA), expression of U6 was set as reference gene. Other gene expressions were performed with SYBR-Green mastermix Kit (Bio-Rad, CA, USA) with $\beta$-actin used as reference gene. All qRTPCR reactions were analyzed for 40 cycles with the ABI 7900HT Fast Real-Time PCR System (Thermo Scientific, MA, USA). Primers were shown as following. miR-21: F: 5'-ACACTCCAGCTGGGTGTAAACATCCTAC ACTCT-3', R: 5'-CTCAACTGGTGTCGTGGA-3'; CDK6: F 5'-TGGAGACCTTCGAGCACC-3', R: 5 ' - CAC TCCAGGCTC TGGAAC T T- 3 '; U6: F: 5'-CTCGCTTCGGCAGCACA-3'; R: 5 '-AACGCTTCACGAATTTGCGT-3'; $\beta$-actin: F: 5 '-CAAGAGATGGCCACGGCTGCT-3', R : 5'-TCCTTCTGCATCCTGTCGGCA-3'.

The $2^{-\Delta \Delta \mathrm{Ct}}$ method was analyzed to explore the expression of target genes.

\section{Western blot (WB) analysis}

Mice renal samples and HK-2 cells were extracted and quantified using the Pierce BCA Protein Assay Kit (Thermo Scientific, MA, USA). Total proteins were denatured using $5 \times$ loading buffer, and then was separated on $10 \%$ SDS-PAGE, next, the protein was transferred to PVDF membrane, which was blocked by PBS containing 3\% BSA and $0.1 \%$ sodium azide for 1 hours at $25^{\circ} \mathrm{C}$. the membranes were incubated with primary antibodies of Bcl-2, Bax, CDK 6 and $\beta$-actin at $4{ }^{\circ} \mathrm{C}$ overnight. Then, then membranes were incubated with horseradish peroxidase (HRP)conjugated secondary antibody at $25^{\circ} \mathrm{C}$ for $1 \mathrm{~h}$. Protein band detection was performed with the help of an enhanced chemiluminescence system.

\section{The miR-target gene interactions}

Seven databases were used to explore the target genes of miR-21 including the Targetscan (18), RNAhybrid (19), Rna22 (20), PicTar5 (21), miRBase (22), Miranda (23), and DIANA-miR (24) databases, and 120 target genes of miR-21 were primarily screened. To investigate function annotation and pathway enrichment of the targets of miR21, Database for Annotation, Visualization, and Integrated Discovery (DAVID) was used to conduct the Kyoto Encyclopedia of Genes and Genomes (KEGG) analysis. 
The statistically significant threshold was considered as a $\mathrm{P}$ value $<0.05$.

\section{Luciferase reporter assay}

The wild-type miR-21 binding site on CDK6 (CDK6WT) and site mutant sequence (CDK6 -Mut) were cloned into the pMIR-REPORT luciferase vector (Ambion, USA). Next, 6-well plates were used to seed HK-2 cells that were later subjected to transfection for $48 \mathrm{~h}$ with the indicated components with lipofectamine 2000 (Invitrogen, USA). Luciferase activity was determined with the Dual-Luciferase reporter 1000 Assay System (Promega, USA).

\section{Caspase-3 activity assay}

A caspase-3 activity assay kit (Beyotime Institute of Biotechnology, Nantong, China) was utilized to quantify caspase-3 activity as per the manufacturer's protocols. Cell lysate supernatants were mixed with a buffer comprising the substrate peptides to enhance caspase-3-pNA binding. ELISA was used to quantify pNA release by reading its absorbance with an ELISA reader at $405 \mathrm{~nm}$. Caspase activity was calculated as the ratio between experimental and control groups.

\section{Plasma miR-21 level in patients with septic AKI}

Evaluation of miR-21's role in sepsis-associated kidney dysfunction was carried out by comparing the miR-2 1 plasma levels between 53 patients with septic AKI and 21 septic patients without AKI. Then, $200 \mu \mathrm{L}$ of plasma was obtained from each patient for total RNA isolation with the mirVana PARIS isolation kit (Ambion, TX, USA) based on the corporation's protocols. The 7900HT Fast RealTime PCR System (Thermo Scientific, MA, USA) was used to quantify miR-21 expression. Informed written consent was obtained from each participant before study commencement. All human investigational procedures were in line with principles, as stated in the Declaration of Helsinki and were approved by the institutional review committee of Harbin Medical University.

\section{HE staining}

Mouse renal tissues were dissected, fixed in $4 \%$ paraformaldehyde (Sigma-Aldrich, MO, USA) for 1 hour at $4{ }^{\circ} \mathrm{C}$, paraffin-embedded, and sectioned into a thickness slice of $5 \mathrm{~mm}$. Following the removal, hematoxylin (Sigma-Aldrich, MO, USA) staining was performed at room temperature for 10 minutes and rinsed using water. Sections were rinsed for 1 minute with Scott Promote Blue Liquid and 20 seconds with $1 \%$ hydrochloric acid alcohol differentiation liquid in turns. Final staining with $0.5 \%$ eosin (Sigma-Aldrich, MO, USA) was then done for 0.5 minutes. Finally, the slices were sealed after washing with purified water.

\section{Statistical analysis}

GraphPad 7.0 Prism Software was used and data are presented as mean \pm SEM. To compare means between 2 subgroups, we used the non-parametric Kruskal-Wallis ANOVA or Student's $t$-test, followed by Dunnett. A $\mathrm{P}<0.05$ or 0.01 was set to be statistically significant.

\section{Results}

miR-21 is increased in patients with septic AKI, HK-2 cells and serum of LPS-stimulated mice

The miR-21 expression in plasma were measured in 53 cases with septic AKI and 21 septic cases without AKI. Markedly increased miR-21 expression in plasma were found in cases with septic AKI (Figure 1A), suggesting that miR-21 elevation in sepsis-associated AKI may be clinically relevant. Moreover, $10 \mathrm{mg} / \mathrm{kg}$ of intraperitoneal LPS injection in mice resulted in increased miR-21 expression in peripheral blood samples based on qRT-PCR analyses (Figure 1B). Comparable results were also noted in HK-2 cells that were given LPS (Figure 1C).

\section{miR-21 inbibition attenuates apoptosis and improves kidney function in $\mathrm{HK}-2$ cells and LPS-stimulated mice}

This research was designed to explore the function of miR21 in LPS-stimulated AKI. Firstly, the miR-21 levels were detected after transfecting with its inhibitor, and the results suggested that the miR-21 inhibitors enormously downregulate its levels (Figure S1). Secondly, we found the cell growth of miR-21 inhibitor + LPS group is up-regulate than the LPS group by MTT assay (Figure 2A). AO/EB staining was firstly used to demonstrate that miR-21 knockdown in LPS-challenged HK-2 cells resulted in reduced apoptosispositive nuclei (Figure $2 B$ ). the data of FCM assay gave the evidence that the knockdown of miR-21 decreased the 

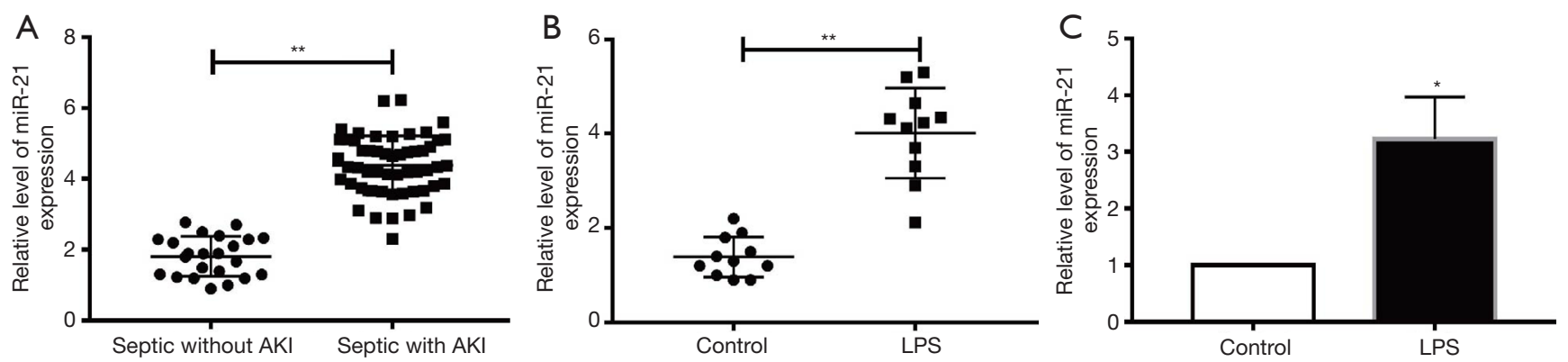

Figure 1 The miR-21 expression level in AKI patients, LPS-stimulated mice, and LPS-stimulated HK-2 cells. (A) The miR-21 level of patients with septic AKI. (B) The peripheral blood miR-21 levels of mice stimulated with LPS. (C) The miR-21 level of HK-2 cells stimulated with LPS. ${ }^{*} \mathrm{P}<0.05$ or ${ }^{* *} \mathrm{P}<0.01$ vs. control group.

apoptosis in LPS-stimulated HK-2 cells (Figure 2C). The WB result suggested that the miR-21 inhibitor upregulated the Bcl-2/Bax ratio (Figure 2D). Furthermore, miR-2 1 inhibitors alleviated LPS-stimulated caspase-3 activity (Figure 2E). Taken together, our data points towards the fact that miR-21 inhibition may improve LPS-stimulated renal cell function and attenuate cellular apoptosis. Creatinine is an important index of AKI (25). We tested the level of creatinine of serum in different groups. Our data showed that miR-21 inhibitor can significantly reduce the level of creatinine induced by LPS (Figure $2 F$ ). HE staining also showed that mice that received miR-21-inhibitor injections for 3 consecutive days before LPS stimulation had better renal function compared to those that did not receive miR21-inhibitor injections (Figure 2G). We also detected the expression of miR-21 in kidney tissues. Our results showed that the expression of miR-21 is down-regulate in miR-21 inhibitor group (Figure $2 \mathrm{H}$ ).

\section{Overexpression of miR-21 aggravates renal dysfunction and apoptosis in HK-2 cells and LPS-stimulated mice}

The miR-21 levels were enormously up-regulated by miR21 mimics in HK-2 cells (Figure S1B). MTT assay was employed to detected the cell growth in each group. The data suggested that the cell viability of miR-21 mimic + LPS group is less than the LPS group (Figure 3A). LPSstimulated HK-2 cells exposed to a miR-21 mimic had increased cellular apoptosis on AO/EB staining (Figure 3B). Similarly, the data of FCM assay suggested that overexpression of miR-21 promoted LPS-stimulated HK-2 cell apoptosis (Figure 3C). Meanwhile, miR-21 mimic upregulated the activity of caspase-3, which was stimulated by LPS in HK-2 cells (Figure 3D), and increased Bcl-2/Bax ratios (Figure 3E) were noted in the LPS-stimulated group. Our results show that miR-21 upregulation can enhance cellular apoptosis after treatment with LPS in HK-2 cells. miR-21 mimic can significantly up-regulate the level of creatinine induced by LPS (Figure $3 F$ ). HE staining also showed that mice that received miR-21-mimic injections for 3 consecutive days before LPS stimulation had worse renal function compared to those that did not receive miR21-mimic injections (Figure 3F). The expression of miR-21 is up-regulate in miR-21 mimic group (Figure 3I).

\section{miR-21 targets the CDK6 gene}

Several bioinformatics tools were performed to show the downstream target of miR-21. We firstly created a miRNAgene interaction by using connections formed in more than 2 of the 7 databases. A total of 120 target genes of miR-21 were obtained (Figure S2). To investigate function annotation and pathways enrichment of the targets of miR21, we used the DAVID to conduct the KEGG analyses which revealed that the cell cycle signaling pathway had an important role (Figure S3, Table 2). miR-21 and CDK6 seed units are shown in Figure 4A. As expected, the CDK6 mRNA level was severely upregulated by miR-21 inhibitor, simultaneously, miR-21 mimics played the opposite role (Figure 4B). Western blot results provided further confirmation that CDK6 represented a functional miR21 target and showed that, after miR-21 mimic treatment, CDK6 protein levels were downregulated (Figure 4C), while the converse was seen after treatment with miR21 inhibitor (Figure 4D). Furthermore, luciferase assay was performed to investigate if CDK6 can directly target to miR-21. Interestingly, cells co-incubated with miR-21 mimic/CDK6-WT displayed reduced luciferase activity. 

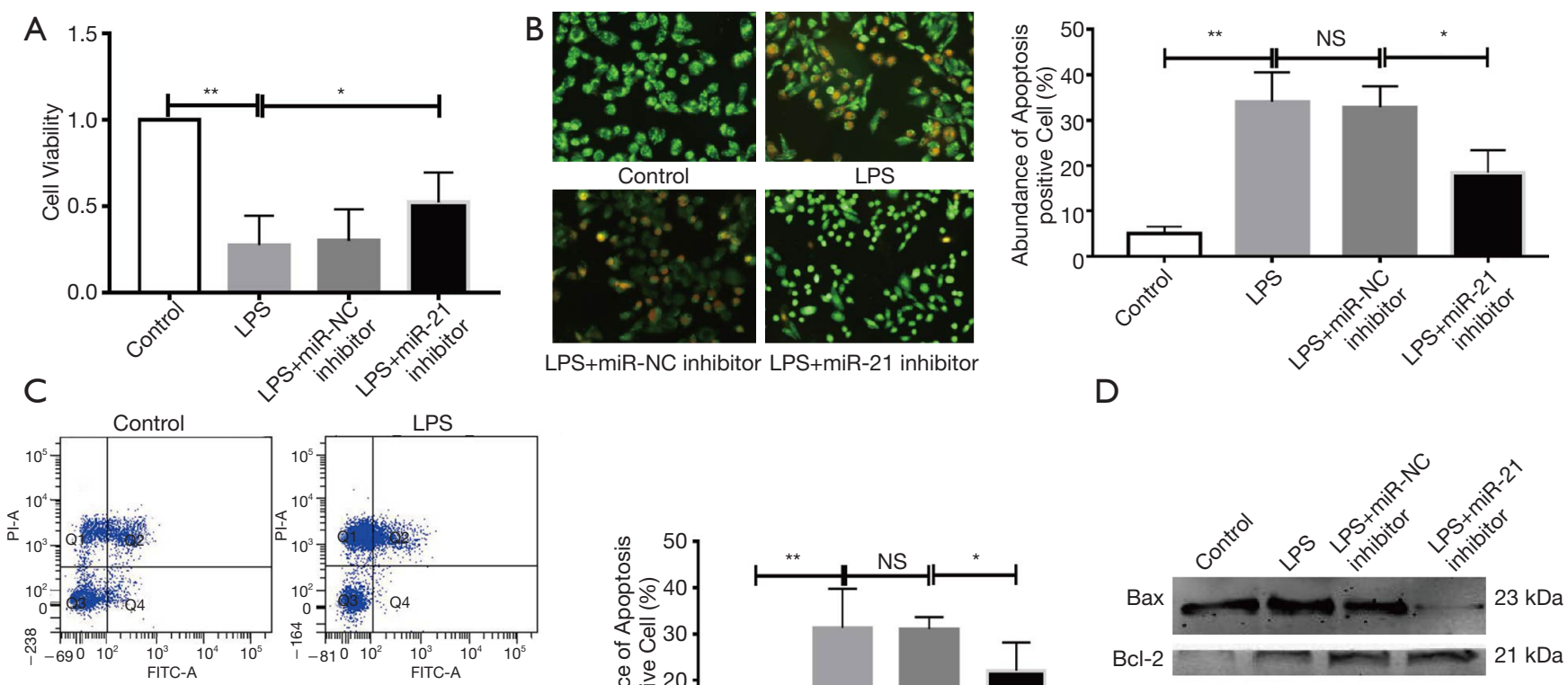

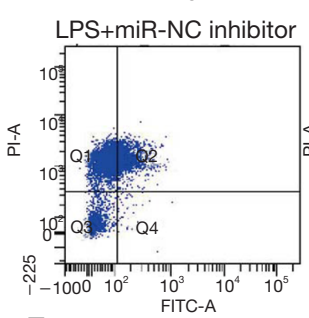

$\mathrm{E}$

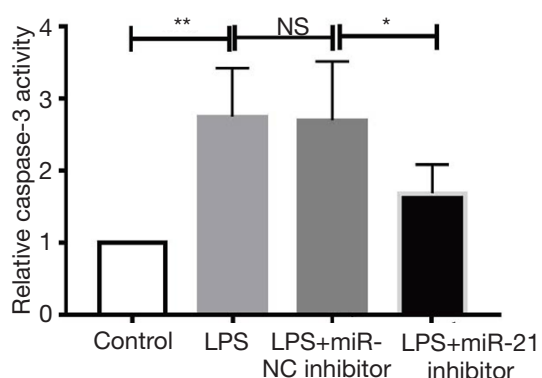

$\mathrm{H}$

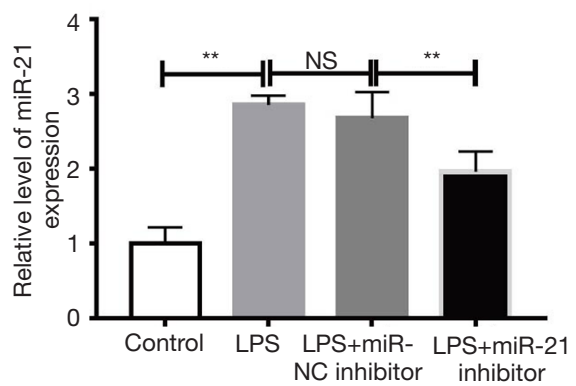

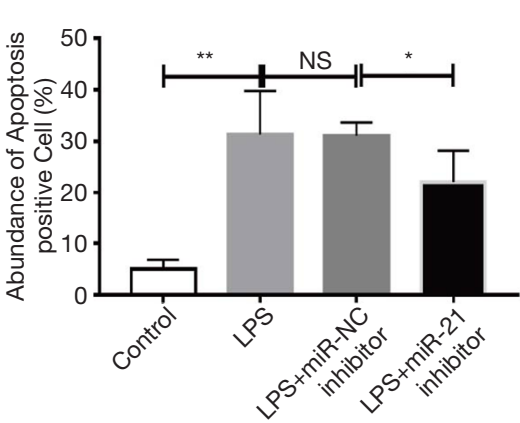

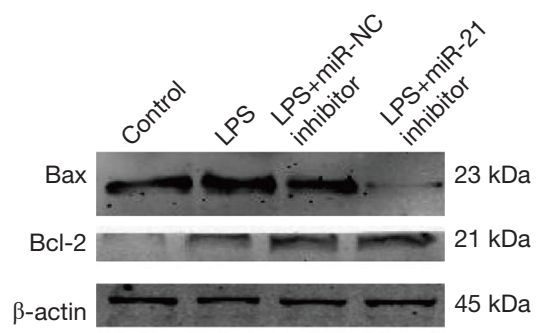

F

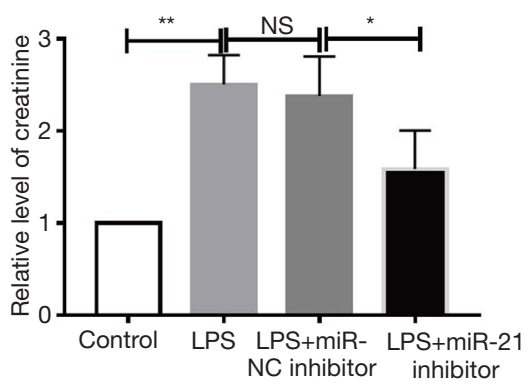

G

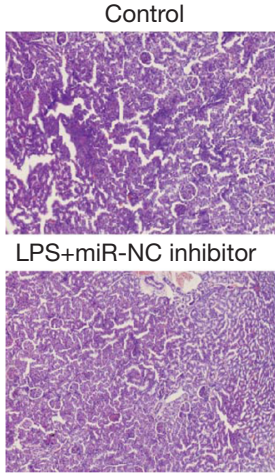

LPS

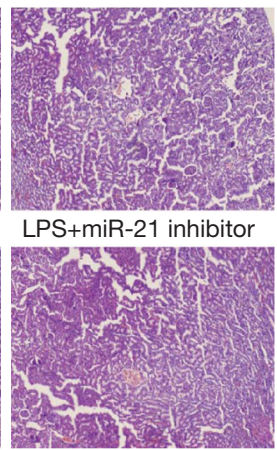

Figure 2 miR-21 inhibitor enhances renal function and abrogates cellular apoptosis in LPS-stimulated mice and human renal tubular cells. HK-2 cells were co-incubated with miR-21 inhibitor or LPS. (A) MTT assay was performed to detected the cell growth; (B) AO/EB staining was employed to detect changes in the nucleus. (C) FCM assay was employed to test the HK-2 cell apoptosis. (D) Western blot for Bax and Bcl-2, and quantitative analysis for Bcl-2/Bax ratio. $\beta$-Actin was used as a reference gene. (E) Caspase-3 activity LPS-stimulated HK-2 cells that were given miR-21 inhibitor or NC group. (F) Relative level of creatinine in serum. (G) HE staining for the renal function. Original magnification was 100x. $\mathrm{HE}$ staining for the renal function. $(\mathrm{H})$ Relative level of miR-2 1 in renal tissue. $n=4,{ }^{*} \mathrm{P}<0.05$ vs. control group or miR-NC inhibitor group or LPS group. ${ }^{*} \mathrm{P}<0.01$ vs. control group or miR-NC inhibitor group or LPS group. $\mathrm{NS}, \mathrm{P}>0.05$ vs. control group. 

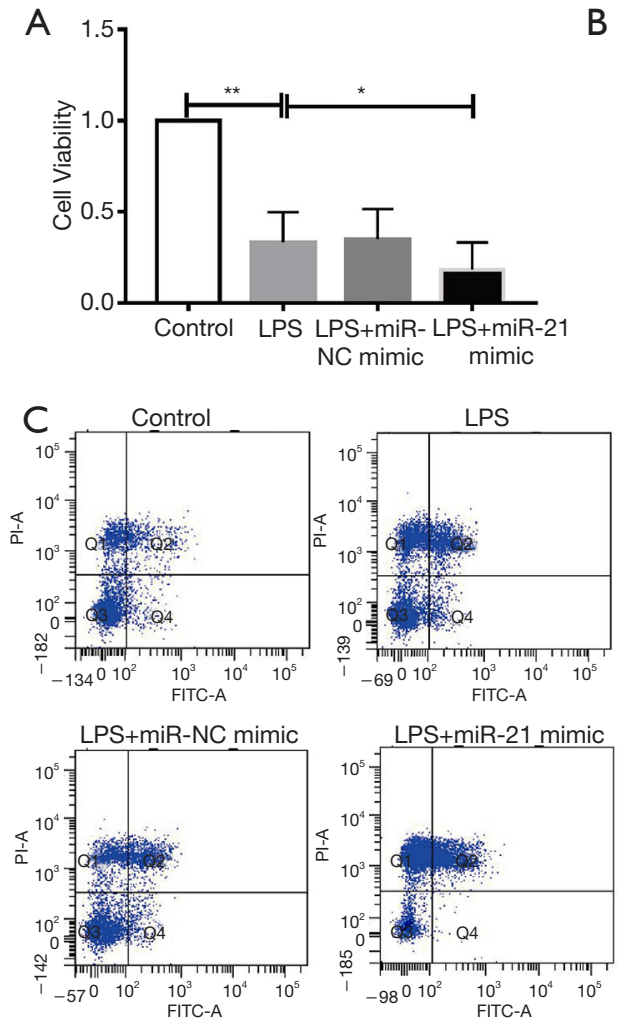

$\mathrm{E}$
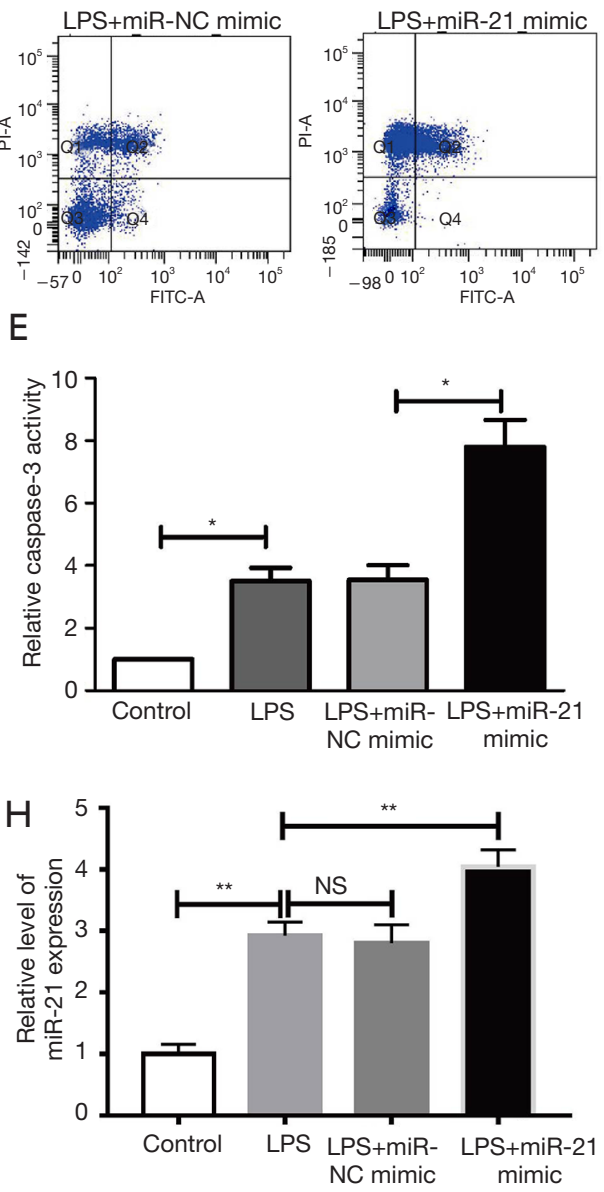

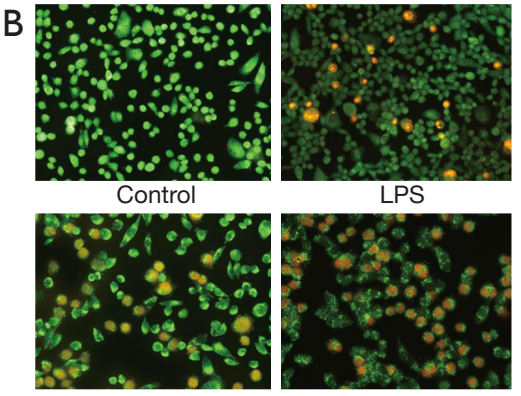

LPS+miR-NC mimic LPS+miR-21 mimic
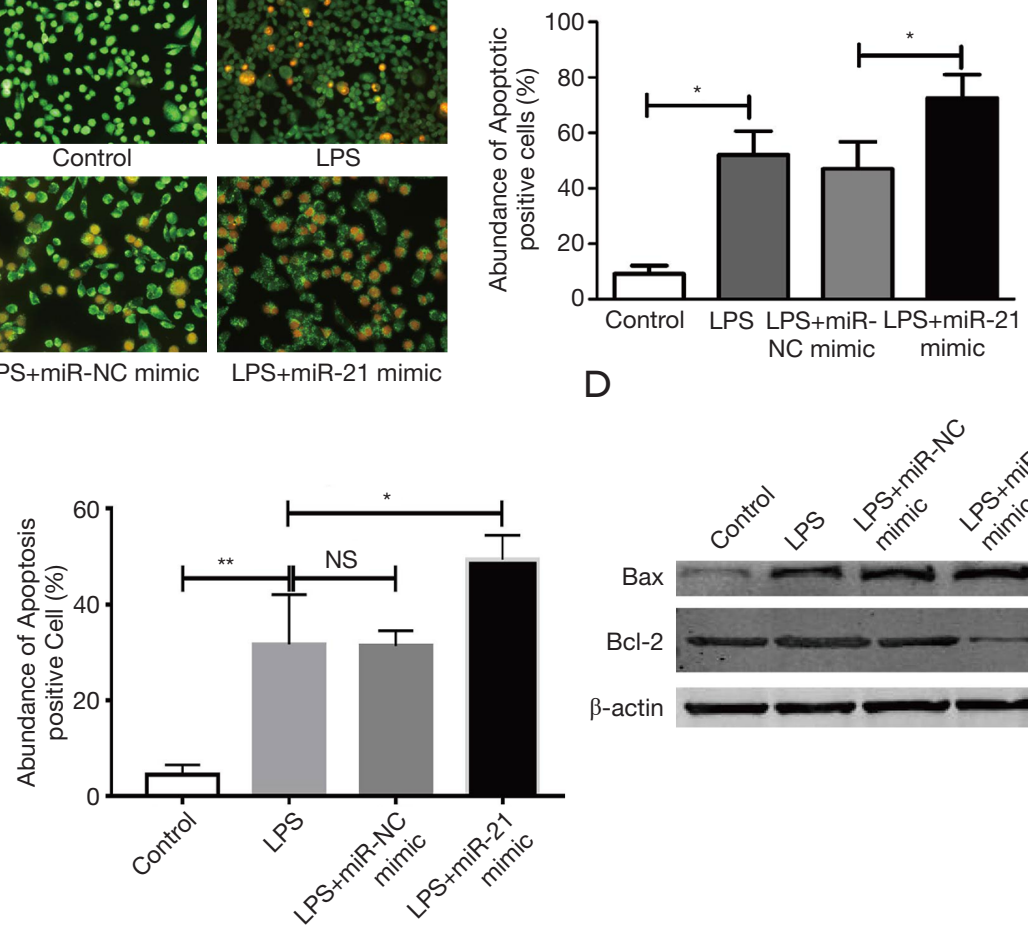

$\mathrm{D}$

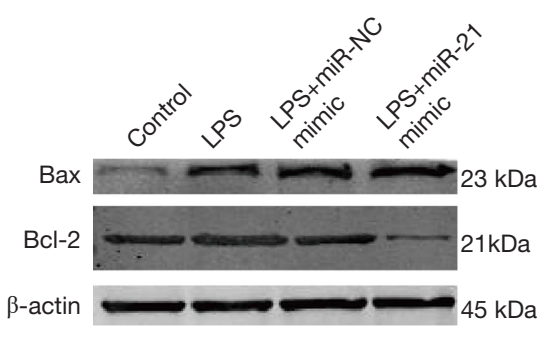

$\mathrm{F}$

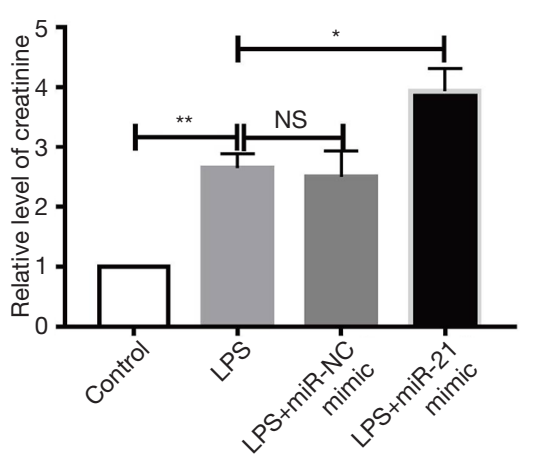

G

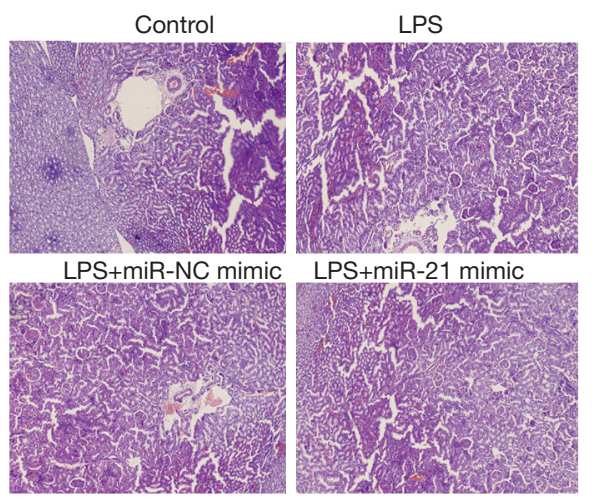

Figure 3 miR-21 mimic further damages renal function and upregulates cellular apoptosis in LPS-stimulated mice and human renal tubular cells. HK-2 cells were co-cultured with miR-21-mimic or LPS. (A) MTT assay. (B) AO/EB staining. (C) FCM assay. (D)WB assay for Bax and Bcl-2, and quantitative analysis for Bcl-2/Bax ratio. (E) Caspase-3 activity. (F) Relative level of creatinine in serum. (G) HE staining for the renal function. Original magnification was $100 \times$. (H) Relative level of miR-21 in renal tissue. $n=4,{ }^{*} \mathrm{P}<0.05 v$ s. control group or miR-NC mimic group or LPS group. ${ }^{* *} \mathrm{P}<0.01$ vs. control group or miR-NC mimic group or LPS group. NS, $\mathrm{P}>0.05$ vs. control group. 
Table 2 Enriched pathways of targets of miR-21

\begin{tabular}{lll}
\hline Term & P value & Genes \\
\hline hsa04110: cell cycle & $1.24 \mathrm{E}-10$ & E2F1, CDC7, CDC6, RBL1, CDK6, SMAD2, RBX1, CDKN1C, \\
& & CCND1, CDKN2A, CCNA2, STAG2, TFDP1 \\
hsa04350: TGF-beta signaling pathway & $3.50 \mathrm{E}-06$ & SMAD7, TGFBR2, RBL1, SMAD2, THBS1, PITX2, RBX1, TFDP1 \\
hsa05212: pancreatic cancer & $1.43 \mathrm{E}-04$ & E2F1, CCND1, CDKN2A, TGFBR2, SMAD2, CDK6 \\
hsa05220: chronic myeloid leukemia & 0.002351 & E2F1, CCND1, CDKN2A, TGFBR2, CDK6 \\
hsa05219: bladder cancer & 0.003949 & E2F1, CCND1, CDKN2A, THBS1 \\
hsa04390: hippo signaling pathway & 0.00635 & CCND1, TGFBR2, SOX2, WWC1, SMAD2, YAP1 \\
hsa05206: microRNAs in cancer & 0.006607 & $R E C K$, E2F1, SPRY2, CCND1, CDKN2A, CDK6, THBS1, PDCD4 \\
hsa05223: non-small cell lung cancer & 0.009453 & E2F1, CCND1, CDKN2A, CDK6 \\
\hline
\end{tabular}

A 5. .....AGACUAAGCCCCCAAAUAAGCUG..... CDK6 WT
3 AGUUGUAGUCAGACUAUUCGAU miR-21-5p
5. AGACUAAGCCCCCAAGUCAAAGG CDK6 Mut

B

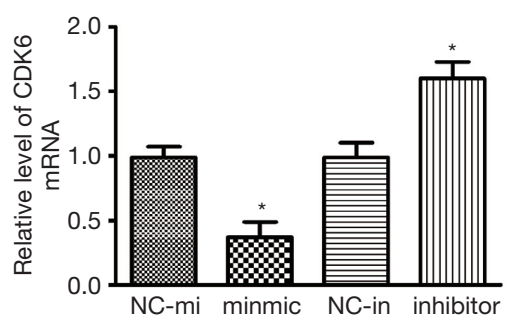

D
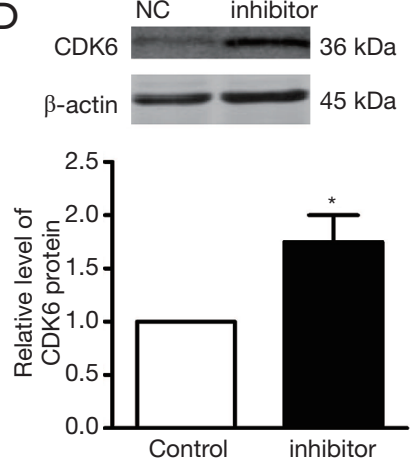

C
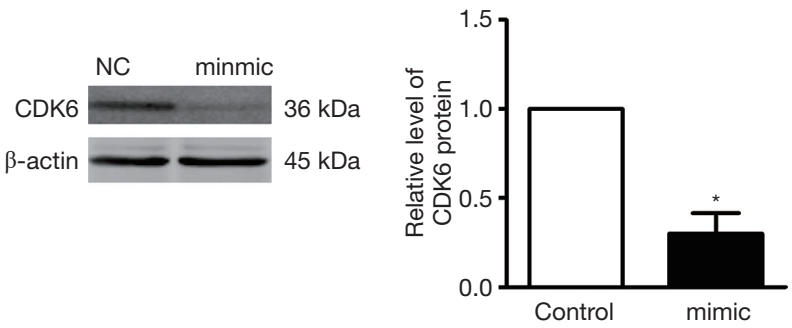

$\mathrm{E}$

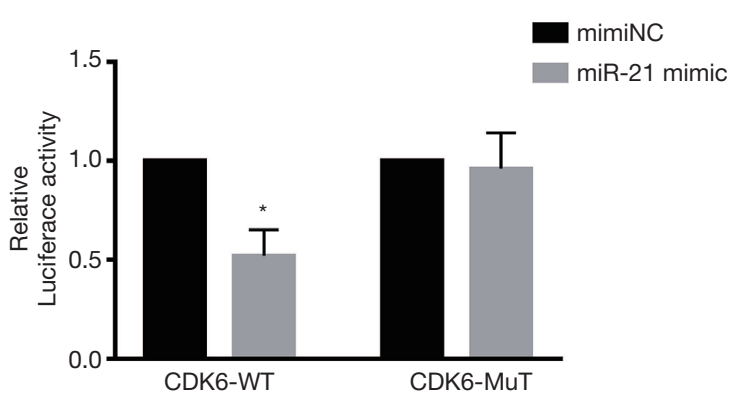

Figure 4 miR-21 directly targets CDK6. (A) Schematic diagram of the miR-21 binding site on CDK6. (B) CDK6 was inversely regulated by miR-21 at the mRNA level (n=3). (C,D) CDK6 was inversely regulated by miR-21 at the protein level (n=4). (E) Luciferase activity. ${ }^{*} \mathrm{P}<0.05$ vs. NC-mi group or NC-in group. 
A
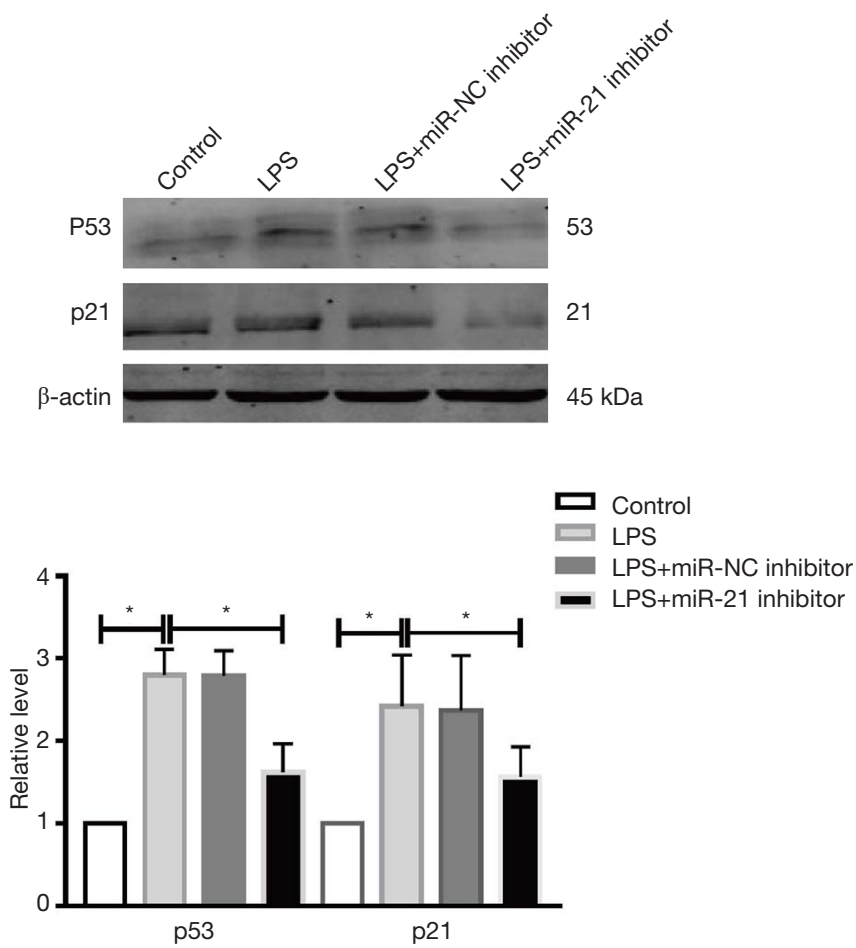

B

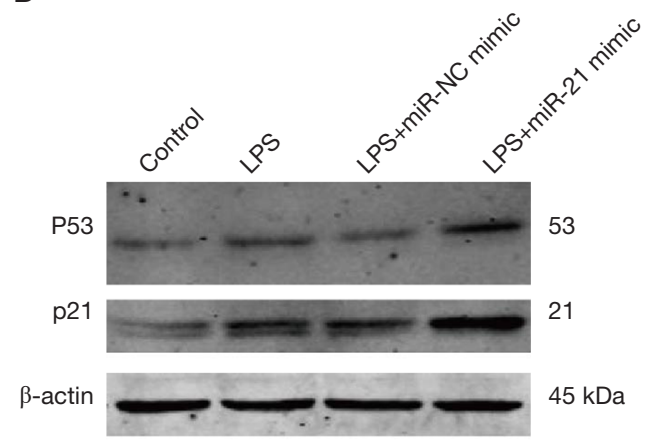

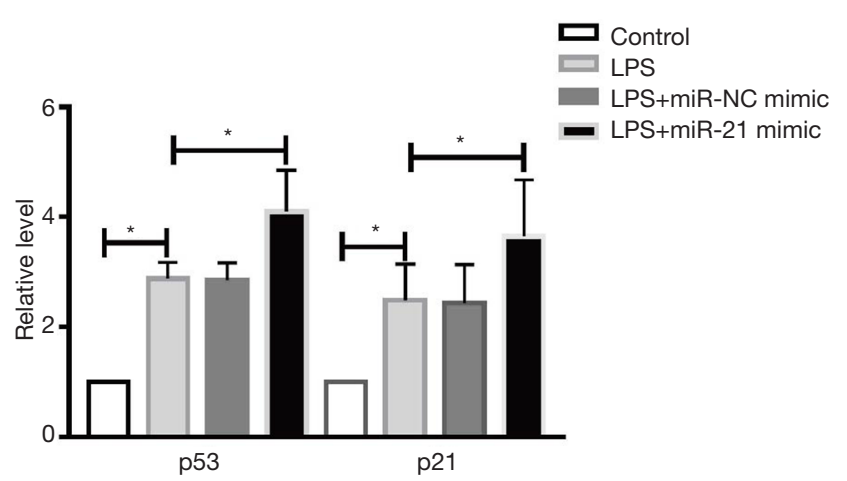

Figure 5 miR-21 regulates p53 and p21 expression caused by LPS in HK- 2 cells. The protein levels of p 53 and p21 after treating with miR21 inhibitor (A) or mimic (B) with LPS. $\mathrm{n}=4,{ }^{*} \mathrm{P}<0.05$ vs. control group or LPS group.

Luciferase activity was undisturbed in the miR-21 mimic/ CDK6 mutant groups (Figure 4E).

\section{Knockdown-miR-21 stimulates $p 53, p 21^{\text {Cip1/Wafl expression }}$ caused by LPS}

Previous work has proved that p53 signaling pathway plays an important role in AKI. WB assay was performed to test the p53 and p21 levels in each group. Our data suggested that the protein levels of p53 and p21 in miR-21 inhibitor group markedly augmented the comet tails compared to the LPS group (Figure $5 A, B$ ). Interestingly, compared with LPS group the miR-21 mimic can upregulated the p53 and p21 levels.

\section{miR-21 attenuates the apoptosis of $\mathrm{HK}-2$ cells and regulates cell functions by inhibiting CDK6}

In order to explore the role of CDK6 during the process of miR-21 involving in LPS-stimulated apoptosis in HK-2 cells, we firstly test the CDK6 levels after LPS injection in vivo and in vitro. In our results CDK-6 is down regulate in peripheral blood samples and HK-2 cells (Figure S4). Then, we used CDK6-siRNA clarify its function (Figure S1C). We co-incubated with miR-21 inhibitor, CDK6-siRNA in LPSstimulated HK-2, and our data suggested that miR-21's negative impact on apoptosis of HK-2 cell were decreased if existing CDK6 (Figure 6). Our data indicate that miR21 has vital effects in LPS-stimulated AKI by promoting CDK6 levels.

\section{Discussion}

Septic AKI is likely to increase the mortality of these critically ill patients (3). Extensive evidence has indicates that several miRs are involved in the process of septic AKI, including miR-212-3p, -122-5p (11), -181a (7), and -20 a (8), although the mechanisms have yet to be fully elucidated. miR-21 is a vital miR related to inflammation, which can be strongly stimulated by LPS both in animal model and cell line (Figure 1). Nevertheless, the details of its pathophysiological mechanisms are still unclear. Our study is unique as it is the first to demonstrate a severe elevation in miR-21 expression in an LPS-damaged kidney. 
Page 10 of 12

A

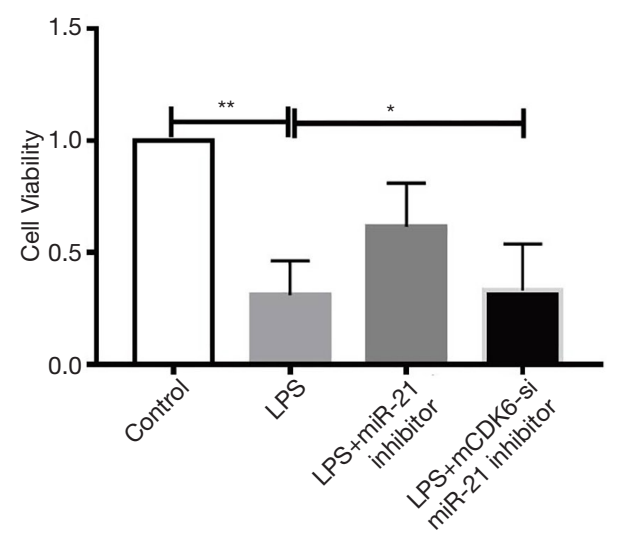

C
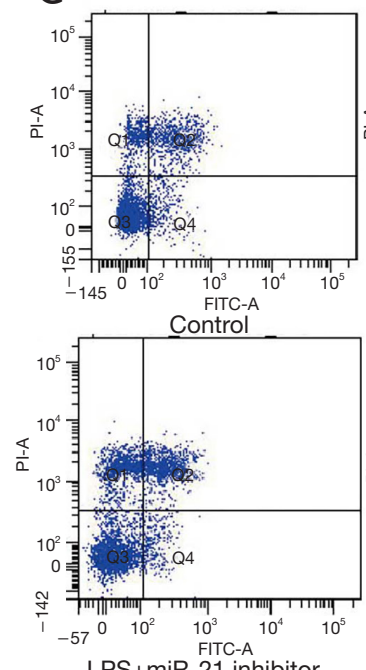

LPS+miR-21 inhibitor

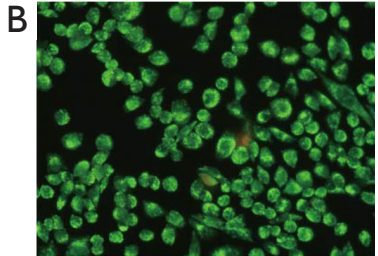

Control

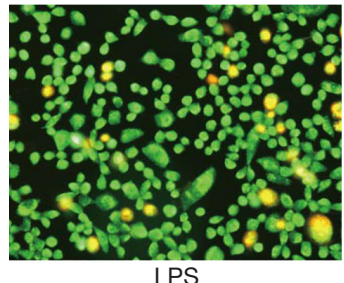

LPS
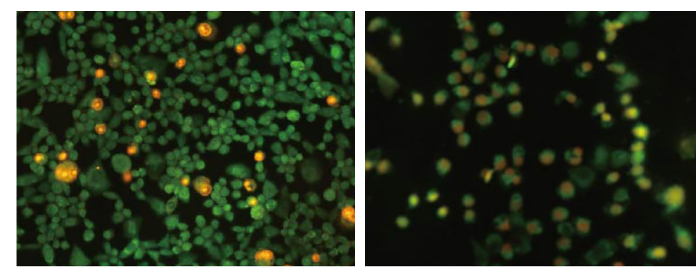

LPS+miR-21 inhibitor

LPS+miR-21 inhibitor+CDK6-si

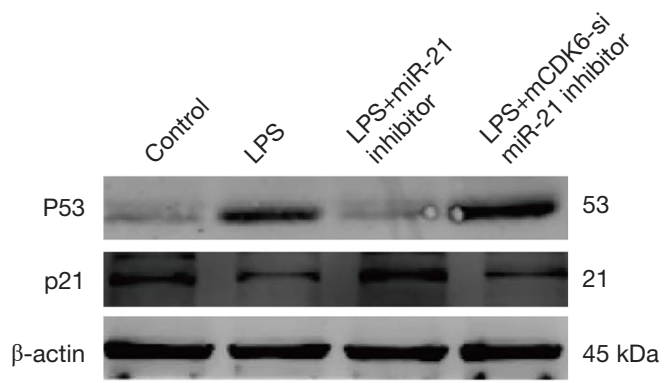

Figure 6 miR-21 attenuated HK-2 apoptosis and was found to regulate their functions by inhibiting CDK6 expression. HK-2 cells were cocultured with miR-21 inhibitor or miR-21 inhibitor+CDK6-siRNA or LPS. (A) MTT assay; (B) AO/EB staining; (C) FCM assay; (D) WB assay for Bax and Bcl-2, and quantitative analysis for Bcl-2/Bax ratio. $\beta$-Actin was used as a reference gene ( $\mathrm{n}=4)$. *, $\mathrm{P}<0.05$ vs. LPS group; **, $\mathrm{P}<0.01$ vs. Control group.

Additionally, we proved that patients with septic AKI had significant increases in plasma miR-21 expression levels. This suggests that miR-21 may be of clinical relevance in septic AKI. In contrast, other reports have found that inducing miR-21 expression reduces renal damage in some abnormal conditions like acute viral renal infections and atherosclerosis. Further studies are needed to substantiate and clarify current evidence surrounding miR-21 functions in septic AKI. Interestingly, miR-21 inhibitors that were co-administered with LPS resulted in attenuated HK-2 cell damage both in animal model and cell line (Figures 2,3). Conversely, over-expressing miR-21 aggravated HK-2 cell damage. This shows that miR-21 may be detrimental to the kidney in sepsis. Esmatabadi et al. found the evidence that miR-21 upregulation decreased the chemotherapeutic effects of endosomal curcumin when administered to breast cancer cell lines (26). Cui et al. also showed miR-21 played a key role in pituitary tumor cells. In our data, we found up-regulate expression of miR-21 enhance the cytotoxicity induced by LPS (Figure 2). Otherwise, knockdown miR-21 expression inhibits the cytotoxicity induced LPS in HK-2 cell (Figure 3). In vivo study, we also found miR-21 mimic decreased the Scr level induced by LPS and kidney injury, while miR-21 inhibitor can reverse it. These data showed miR-21 play an important role in AKI both in vivo and vitro. Moreover, p53 pathway acts a vital role in cell apoptosis, 
which is highly involved in sepsis $(27,28)$. Our study further reveals that LPS-stimulated apoptosis was attenuated with miR-21 inhibition, as shown by in vitro and in vivo evidence of reduced apoptosis-positive nuclei and upregulated Bcl$2 / \mathrm{Bax}$ ratio, and the protein determinations of p53 and p21 while up-regulation of miR-21 conferred contrasting effects. Based on this data, miR-21 may be targeted in developing therapies to reduce cellular apoptosis and p53 signaling pathway during sepsis (Figure 5). Bioinformatics prediction singled out CDK6 as a putative miR-21 target gene. Therefore, a further examination of CDK6 expression levels in LPS- stimulated kidney injury was undertaken. In short CDK6 was confirmed to be down-regulated in LPSstimulated HK-2 cells, with miR-21 acting as a negative regulation factor in vivo. In our study, we found the protein level of CDK6 was downregulated after treatment with LPS. miR-21 inhibitor promotes CDK6 mRNA level downregulation, while miR-21-mimic plays the opposite effect. Comparable results were reported in the luciferase analysis. Taken together, we can conclude that CDK6 can directly target to miR-21. The CDK6 has been documented to contribute towards a myriad of biological processes and participates in regulating neurodegenerative diseases and cancers, but its role in renal illness is still unclear. In our data, we found co-cultured with the miR-21 inhibitor, CDK6-siRNA, or in miR-21 inhibitor + CDK6-siRNA could significantly reverse the function by miR-2 1 inhibitor alone. Our data give support for the notion that miR21 directly targets the CDK6 gene leading to septic renal dysfunction by inducing cellular apoptosis, thus underlying that the miR-21/CDK-6 signal may be a viable indictor in treating septic renal dysfunctions.

\section{Conclusions}

In conclusion, we demonstrate that miR-21 is increased in septic AKI, and that pharmacologically inhibiting miR-21 improves renal function and reduces apoptosis in HK-2 or septic AKI. Therefore, the miR-21/CDK6 signal might be a valuable marker in septic AKI.

\section{Acknowledgments}

Funding: This study was supported by Harbin Medical University Scientific Research 295 Innovation Fund (No.2017LCZX115) and Project of Heilongjiang Provincial Youth Science Foundation (QC2018099).

\section{Footnote}

Conflicts of Interest: The authors have no conflicts of interest to declare.

Ethical Statement: The authors are accountable for all aspects of the work in ensuring that questions related to the accuracy or integrity of any part of the work are appropriately investigated and resolved. Ethical approval was obtained from the Science and Technology Department of Heilongjiang Province. All animals were raised based on the Provisions and General Recommendation of the Chinese Experimental Animals Administration Legislation.

Open Access Statement: This is an Open Access article distributed in accordance with the Creative Commons Attribution-NonCommercial-NoDerivs 4.0 International License (CC BY-NC-ND 4.0), which permits the noncommercial replication and distribution of the article with the strict proviso that no changes or edits are made and the original work is properly cited (including links to both the formal publication through the relevant DOI and the license). See: https://creativecommons.org/licenses/by-nc-nd/4.0/.

\section{References}

1. Lin $\mathrm{S}$, Jin $\mathrm{P}$, Shao C, et al. Lidocaine attenuates lipopolysaccharide-induced inflammatory responses and protects against endotoxemia in mice by suppressing HIF1alpha-induced glycolysis. Int Immunopharmacol 2020;80:106150.

2. Prowle JR. Sepsis-Associated AKI. Clin J Am Soc Nephrol 2018;13:339-42.

3. Antal O, Stefanescu E, Mlesnite M, et al. Hemodynamic Predictors for Sepsis-Induced Acute Kidney Injury: A Preliminary Study. J Clin Med 2020. doi: 10.3390/ jcm9010151.

4. Shimokawa T, Yoneda K, Yamagata M, et al. Yohimbine ameliorates lipopolysaccharide-induced acute kidney injury in rats. Eur J Pharmacol 2020;871:172917.

5. Stanski NL, Stenson EK, Cvijanovich NZ, et al. PERSEVERE Biomarkers Predict Severe Acute Kidney Injury and Renal Recovery in Pediatric Septic Shock. Am J Respir Crit Care Med 2020. [Epub ahead of print].

6. Roka B, Tod P, Kaucsar T, et al. The Acute Phase Response Is a Prominent Renal Proteome Change in Sepsis in Mice. Int J Mol Sci 2019. doi: 10.3390/ijms21010200. 
7. Wang J, Song J, Li Y, et al. Down-regulation of LncRNA CRNDE aggravates kidney injury via increasing MiR181a-5p in sepsis. Int Immunopharmacol 2019;79:105933.

8. Zhang L, He S, Wang Y, et al. miRNA-20a suppressed lipopolysaccharide-induced $\mathrm{HK}-2$ cells injury via NFkappaB and ERK1/2 signaling by targeting CXCL12. Mol Immunol 2020;118:117-23.

9. Pan T, Jia P, Chen N, et al. Delayed Remote Ischemic Preconditioning ConfersRenoprotection against Septic Acute Kidney Injury via Exosomal miR-21. Theranostics 2019;9:405-23.

10. Chen W, Ruan Y, Zhao S, et al. MicroRNA-205 inhibits the apoptosis of renal tubular epithelial cells via the PTEN/Akt pathway in renal ischemia-reperfusion injury. Am J Transl Res 2019;11:7364-75.

11. Cheng Q, Wang L. LncRNA XIST serves as a ceRNA to regulate the expression of ASF1A, BRWD1M, and PFKFB2 in kidney transplant acute kidney injury via sponging hsa-miR-212-3p and hsa-miR-122-5p. Cell Cycle 2020;19:290-9.

12. Zhang XB, Chen X, Li DJ, et al. Inhibition of miR-155 Ameliorates Acute Kidney Injury by Apoptosis Involving the Regulation on TCF4/Wnt/beta-Catenin Pathway. Nephron 2019;143:135-47.

13. Cheng X, Zhang G, Zhang L, et al. Mesenchymal stem cells deliver exogenous miR-21 via exosomes to inhibit nucleus pulposus cell apoptosis and reduce intervertebral disc degeneration. J Cell Mol Med 2018;22:261-76.

14. Feng MG, Liu CF, Chen L, et al. MiR-21 attenuates apoptosis-triggered by amyloid-beta via modulating PDCD4/ PI3K/AKT/GSK-3beta pathway in SH-SY5Y cells. Biomed Pharmacother 2018;101:1003-7.

15. Zhang Y, Xiao Y, Ma Y, et al. ROS-mediated miR-21$5 \mathrm{p}$ regulates the proliferation and apoptosis of $\mathrm{Cr}(\mathrm{VI})-$ exposed L02 hepatocytes via targeting PDCD4. Ecotoxicol Environ Saf 2020;191:110160.

16. Song N, Zhang T, Xu X, et al. miR-21 Protects Against Ischemia/Reperfusion-Induced Acute Kidney Injury by Preventing Epithelial Cell Apoptosis and Inhibiting Dendritic Cell Maturation. Front Physiol 2018;9:790.

Cite this article as: Wei W, Yao YY, Bi HY, Zhai Z, Gao Y. miR-21 protects against lipopolysaccharide-stimulated acute kidney injury and apoptosis by targeting CDK6. Ann Transl Med 2020;8(6):303. doi: 10.21037/atm.2020.03.01
17. Venkatesan S, Rideout JM, Simpson KJ. Microsomal delta 9 , delta 6 and delta 5 desaturase activities and liver membrane fatty acid profiles in alcohol-fed rats. Biomed Chromatogr 1990;4:234-8.

18. Lewis BP, Shih IH, Jones-Rhoades MW, et al. Prediction of mammalian microRNA targets. Cell 2003;115:787-98.

19. Rehmsmeier M, Steffen P, Hochsmann M, et al. Fast and effective prediction of microRNA/target duplexes. RNA 2004;10:1507-17.

20. Miranda KC, Huynh T, Tay Y, et al. A pattern-based method for the identification of MicroRNA binding sites and their corresponding heteroduplexes. Cell 2006;126:1203-17.

21. Krek A, Grun D, Poy MN, et al. Combinatorial microRNA target predictions. Nat Genet 2005;37:495-500.

22. Kozomara A, Griffiths-Jones S. miRBase: integrating microRNA annotation and deep-sequencing data. Nucleic Acids Res 2011;39:D152-7.

23. Betel D, Wilson M, Gabow A, et al. The microRNA. org resource: targets and expression. Nucleic Acids Res 2008;36:D149-53.

24. Maragkakis M, Vergoulis T, Alexiou P, et al. DIANAmicroT Web server upgrade supports Fly and Worm miRNA target prediction and bibliographic miRNA to disease association. Nucleic Acids Res 2011;39:W145-8.

25. Moledina DG, Parikh CR. Phenotyping of Acute Kidney Injury: Beyond Serum Creatinine. Semin Nephrol 2018;38:3-11.

26. Esmatabadi MJD, Farhangi B, Montazeri M, et al. Upregulation of miR-21 decreases chemotherapeutic effect of dendrosomal curcumin in breast cancer cells. Iran J Basic Med Sci 2017;20:350-9.

27. He L, Wei Q, Liu J, et al. AKI on CKD: heightened injury, suppressed repair, and the underlying mechanisms. Kidney Int 2017;92:1071-83.

28. Peng J, Li X, Zhang D, et al. Hyperglycemia, p53, and mitochondrial pathway of apoptosis are involved in the susceptibility of diabetic models to ischemic acute kidney injury. Kidney Int 2015;87:137-50. 

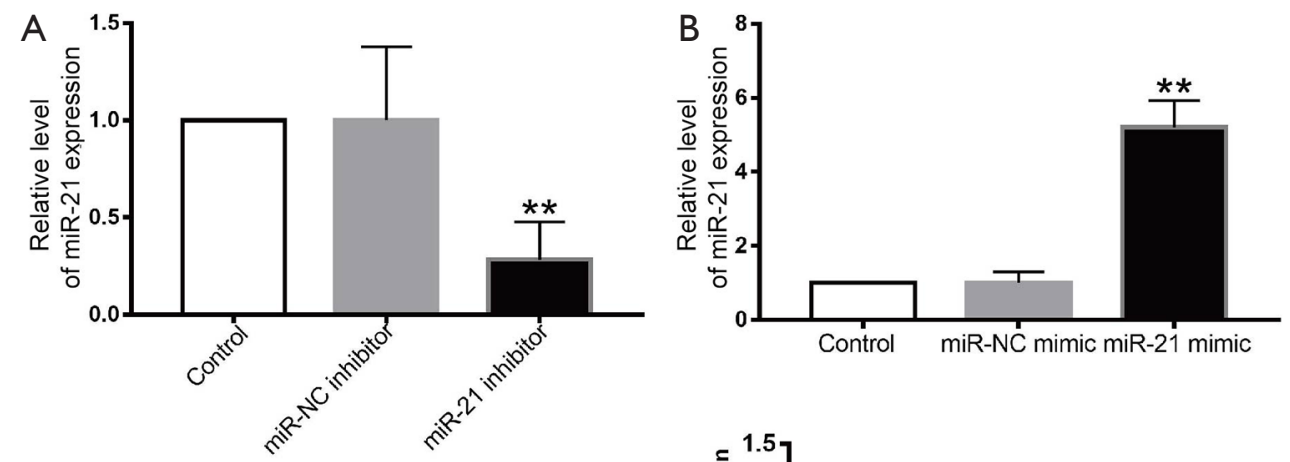

C
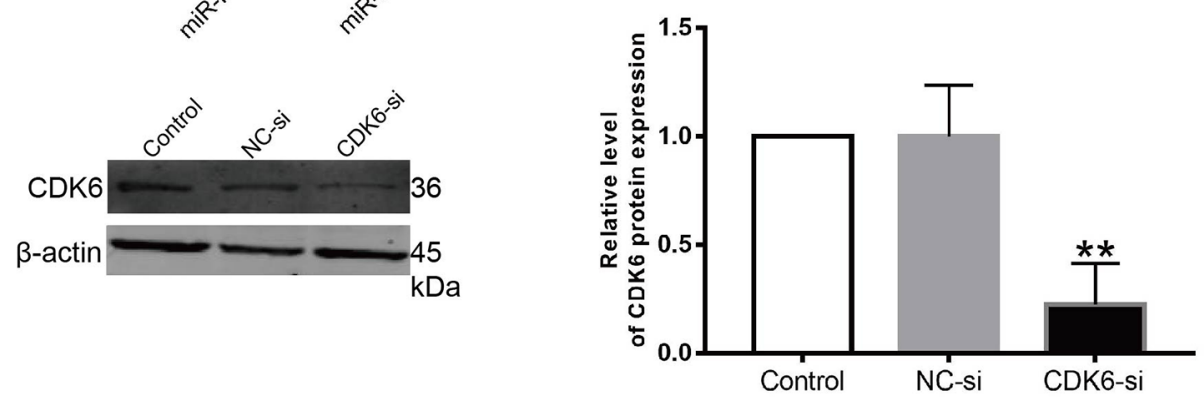

Figure S1 The expression of CDK6 after co-cultured with CDK6-siRNA. (A) Relative level of miR-21 after transfect with miR-21 inhibitor; (B) relative level of miR-21 after transfect with miR-21 mimic; (C) relative level of CDK6 after transfect with CDK6-siRNA. **, $\mathrm{P}<0.01$ vs. control group or miR-NC mimic group or miR-NC inhibitor group. 


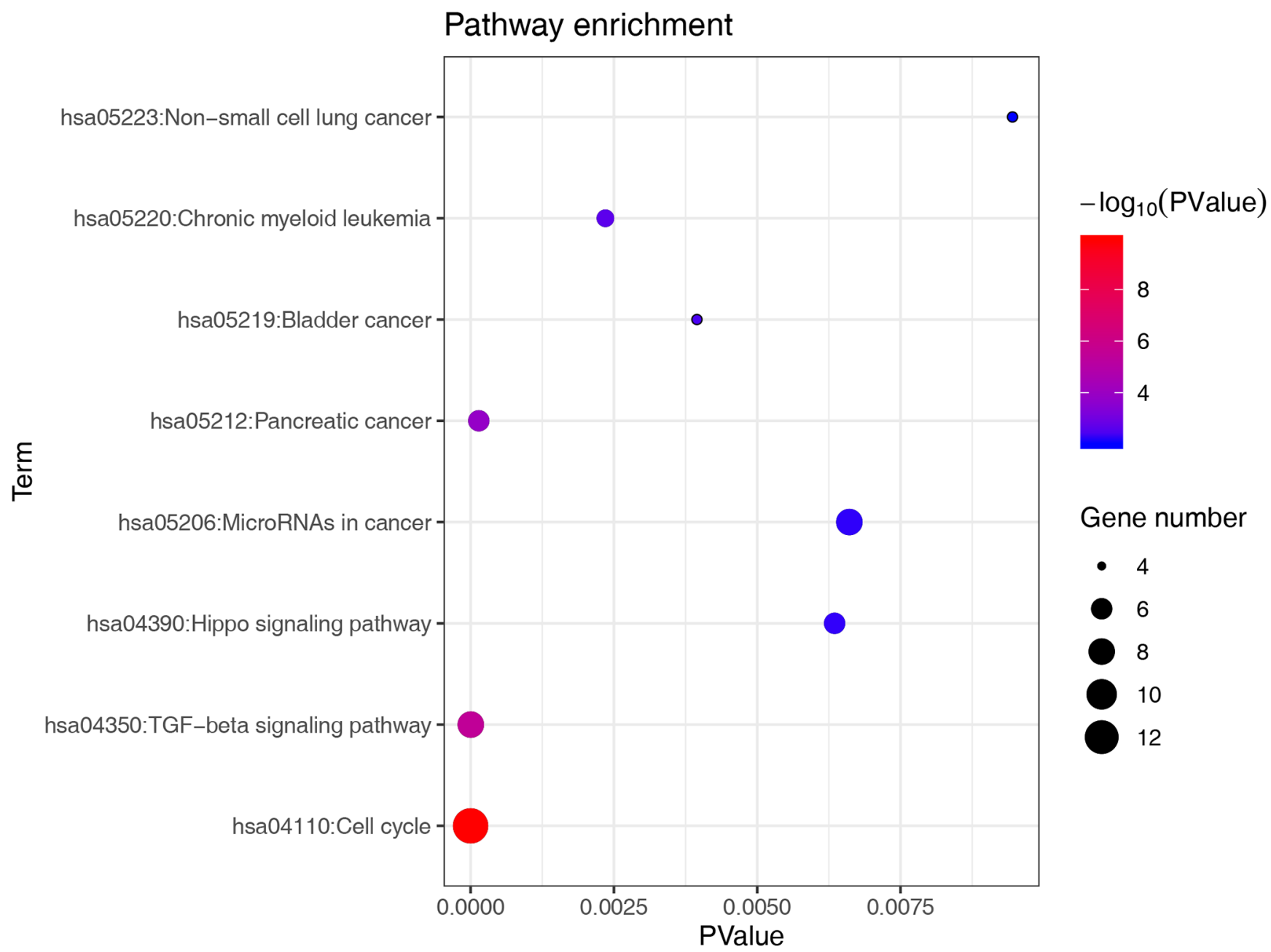

Figure S2 Target genes of miR-21 in AKI. 


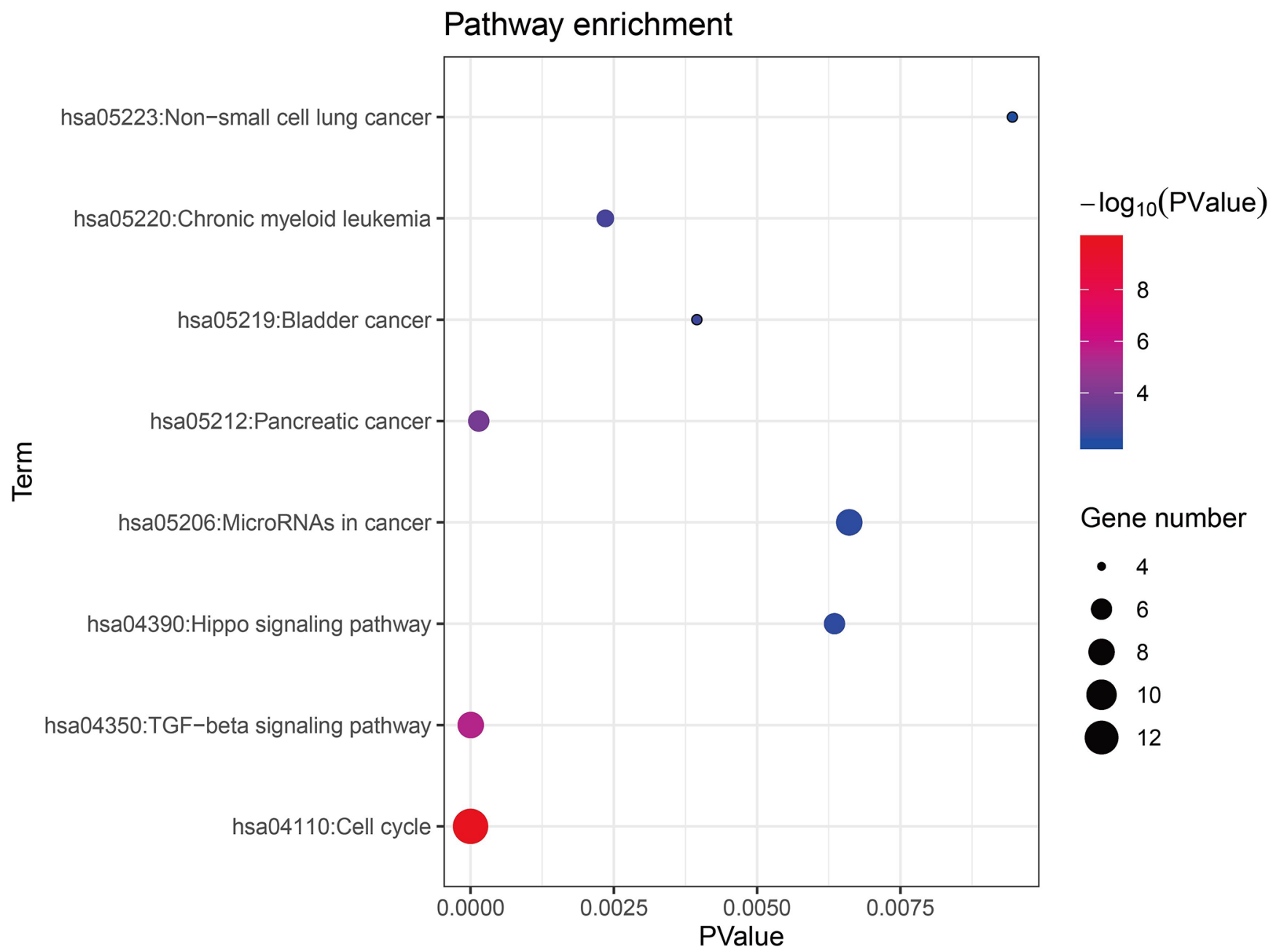

Figure S3 miR-21 regulate signaling pathways in AKI. 

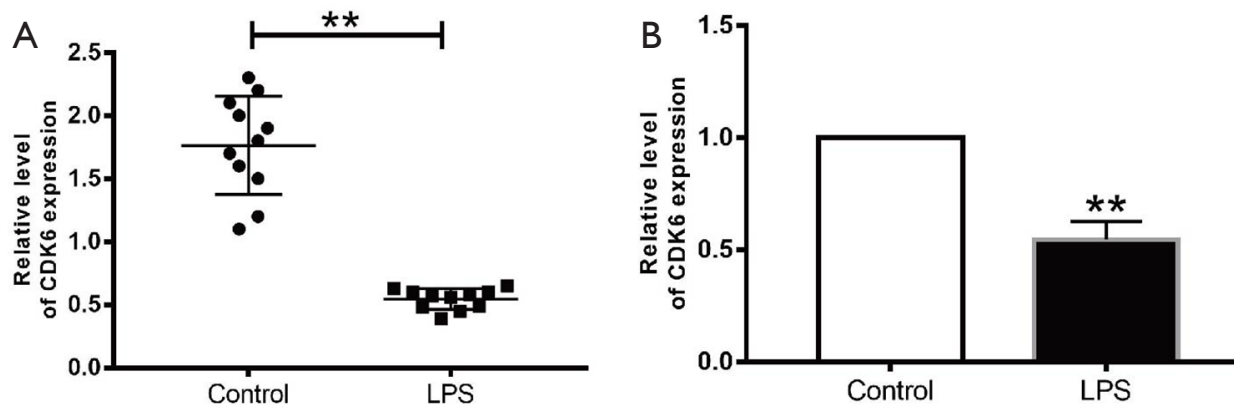

Figure S4 The expression of CDK-6 in AKI mouse and HK2 cells by LPS. (A) The expression of CDK-6 in AKI mouse; (B) the expression of CDK-6 in HK2 cells by LPS. **, $\mathrm{P}<0.01$ vs. control group. 